\title{
USE OF MUSIC DURING PHYSICAL THERAPY INTERVENTION IN A NEONATAL INTENSIVE CARE UNIT: A RANDOMIZED CONTROLLED TRIAL
}

\author{
Cristiane Aparecida Moran', Roberta de Oliveira Cacho', Enio Walker Azevedo Cacho², \\ Klayton Galante Sousa', Jane Carla de Souza², Gentil Gomes da Fonseca Filho ${ }^{3}$, \\ Silvana Alves Pereira ${ }^{4}$
}

DOI: http://dx.doi.org/10.7322/jhgd.103004

\begin{abstract}
Introduction: Previous studies have shown music therapy as a way to improve the clinical status of premature infants, improving vital signs and decreasing crying episodes. Objective: Compare the effects of music on vital signs of premature infants undergoing respiratory physiotherapy. Methods: We performed a randomized clinical trial consisting of 26 premature infants, divided into control group $(\mathrm{N}=12)$ and study group $(\mathrm{N}=14)$, carried out in a Neonatal Intensive Care Unit. All newborn infants undergone standard practice physiotherapy for 15 minutes: vibration and aspiration. On the study group they were exposed to classical music three minutes before the standard physiotherapy, and finished three minutes after the end of these procedures. A recorder was placed inside the incubator with an intensity of 25 decibels. Measurements of heart and respiratory rate and oxygen saturation were measured before, during and after each sessions of respiratory physiotherapy for two groups. Results: When comparing control and study groups regarding heart rate frequency and oxygen saturation, there was no statistically significant difference, but the inclusion of music determined clinically the variability of these data and $30 \%$ of the variation in respiratory rate was due to the inclusion of music during and after the physiotherapy, and the neonatal noninvasive ventilation showed less variation in their respiratory rate when compared to the neonatal on oxygen therapy. Conclusion: The results have showed a beneficial effect of music, showing lower respiratory rate in newborn infants during and after respiratory physiotherapy procedure.
\end{abstract}

Key words: acoustic stimulation, therapy, complementary therapies, newborn, underweight baby.

\section{INTRODUCTION}

Music enhances the human expression ${ }^{1}$ and in its universality has been played an important role in the social relations ${ }^{2}$, being suggested as a resource for health improvement ${ }^{3}$.

Songs with more serene rhythms and melodies, calm and simple harmony are suggested to provide feelings of tranquility ${ }^{4}$ and physiological stability ${ }^{1,5}$. This more relaxed state leads to decrease heart and respiration rates, anxiety and agitation ${ }^{6,7}$. These characteristics have encourage the use of music in hospitals, especially in neonatal intensive care unit ${ }^{2,7,8}$. Music therapy is defined as the prescription of music and the musical interventions to restore, maintain and improve newborn's emotional standpoint, physiological and your wellness ${ }^{8}$. Studies show music therapy as a way to promote improvement of clinical and social status in preterm newborns, improving, among other conditions, heart and respiratory rate, level of oxygen saturation, decreasing crying episodes ${ }^{9,10}$ and thus, promoting quality of sleep ${ }^{11}$.

However when hospitalized in a Neonatal Intensive Care Unit while receiving care that optimize their chances of survival, these newborns often do not receive appropriate interactions for their clinical and social development. Therapies such as the respiratory physiotherapy even though intended to reduce complications of prematurity itself and lessen the environmental conditions to which they are exposed can cause changes in physiological and behavioral parameters of preterm infants ${ }^{12,13}$.

Although some studies investigated the behavioral and physiological responses to music on infants ${ }^{1-6}$, only a few published reports supported the use of music with premature infants during respiratory physiotherapy ${ }^{2}$, therefore the objective was to evaluate the effects of music on vital signs of preterm infants undergoing respiratory physiotherapy.

1 PhD by Federal University of Sao Paulo - Unifesp, Sao Paulo, Brazil.

2 PhD and Adjunct Professor at Federal University of Rio Grande do Norte - UFRN/FACISA, RN, Brazil.

3 Physiotherapist, Resident at the University Hospital Ana Bezerra, HUAB/EBSERH/UFRN, RN, Brazil.

4 PhD and Adjunct Professor at Residence at the University Hospital Ana Bezerra and Federal University of Rio Grande do Norte - UFRN/ EBSERH/FACISA, RN, Brazil.

Corresponding author: Silvana Alves Pereira. E-mail: apsilvana@usp.br

Suggested citation: Moran CA, Cacho RO, Cacho EWA, Souza KG, Souza JC, Fonseca Filho GC, et al. Use of music during physical therapy intervention in a neonatal intensive care unit: a randomized controlled trial. Journal of Human Growth and Development. 25(2): 177-181. DOI: http://dx.doi.org/10.7322/jhgd.103004

Manuscript submitted: Oct 22 2014, accepted for publication Dec 192014. 


\section{METHODS}

Between August 2009 to August 2010 was carried out in the Neonatal Intensive Care Unit of the Mandaqui Hospital, Sao Paulo city, Sao Paulo, Brazil a randomized clinical trial with premature newborns that were allocated to the study (respiratory physiotherapy with music) or control (respiratory physiotherapy without music) group. The study started after approval of Ethics Committee in research $\left(n^{\circ}: 248694\right)$ and signing informed consent term by the newborn infant 's parents. The plan was recruit 10 to 15 participants for each group as a sample of convenience representing premature infants receiving respiratory physiotherapy at Neonatal Intensive Care Unit of the Mandaqui Hospital.

To determine the trial allocation between groups was performed a draw with 26 premature infants of low weight, who had medical prescription for physiotherapy and still in the incubator. Two preterm newborn infants with sedative prescription and neurological problems such as periventricular hemorrhage and neonatal anoxia have not participated the draw. All 26 newborn infants were undergone standard practice physiotherapy for 15 minutes: vibration and airways aspiration, of these, 14 were allocated to the study group and exposed to classical music (New Age).

A Panasonic microcassette $\AA$ recorder was placed inside the incubator with an intensity of 25 decibels. The exposure to music started three minutes before the standard physiotherapy, and finished three minutes after the end of these procedures. The physiotherapeutic procedures with or without music were carried out by two researchers previously trained for this function.

And the control group consisted of 12 preterm newborn infants not exposed to music in any time.

In order to evaluate the music therapy effect, the values of heart and respiratory rates, and oxygen saturation were recorded before, during and after the beginning of each physiotherapy session and each premature infant received two sessions.

The measurement of heart rate and oxygen saturation was carried out by a monitoring system of vital signs DX 2010-LCD - Dixtal Boimedical $®$ and the respiratory rate was measured for one minute by a manual stopwatch.

The results are shown as average \pm standard deviation. Statistical analyses were performed with analysis of variance for repeated measures (ANOVA). The Bonferroni test was used for multiple comparisons. We decided by a significant level of á d" 0.05 and consider as null hypothesis that the music therapy does not alter the physiological changes during the physiotherapy procedures.

\section{RESULTS}

Twenty six newborn infants were chosen and $77 \%$ were male. The average weight was $1268 \pm$ $537 \mathrm{~g}$ and the gestational average age was $31 \pm 3$ weeks. The prevalent diseases observed among 26 preterm infants were: respiratory distress syndrome
(73\%), newborn infant jaundice (27\%) newborn infant sepsis (15\%), and pleural effusion (12\%), 15 were on oxygen and 11 on non-invasive mechanical ventilation.

There was no statistically significant difference between groups regarding the prevalence of diseases; $p<0.999$; birth weight $(p=0.837)$ and gestational age $(p>0.999)$.

When comparing control and study groups regarding heart rate frequency and oxygen saturation, there was no statistically significant difference, but the inclusion of music determined clinically the variability of these data and $30 \%$ of the variation in respiratory rate was due to the inclusion of music during and after the physiotherapy ( $F(3.97) ; p=0.03$ ) Figure 1 has shown the result of this analysis.

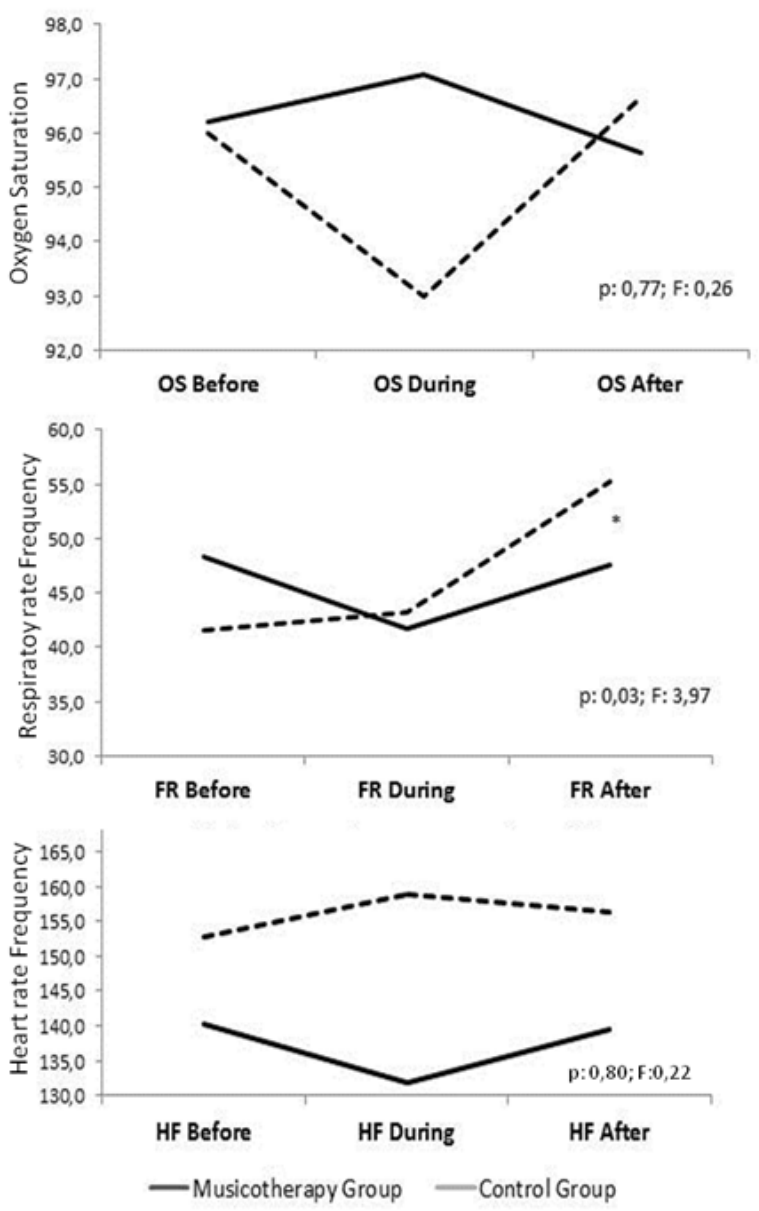

Figure 1: Physiological variables during Physiotherapy Procedures. There was no statistically significant difference when comparing the two control and study groups regarding heart rate frequency and oxygen saturation and $30 \%$ of the variation in respiratory rate was due to the inclusion of music during and after the physiotherapy (F (3.97); p: 0.03); OS: Oxygen Saturation; FR: Respiratpry Frequency; HR: heart rate Frequency.

Another factor that influenced the respiratory rate pattern was the interaction between the music therapy and the ventilation support (oxygen or noninvasive ventilation). In this analysis the respiratory rate variation was lower in newborn of music therapy group with oxygen support ( $F$ (5.06: $p=0.01)$. Figure 2 has shown these results. 


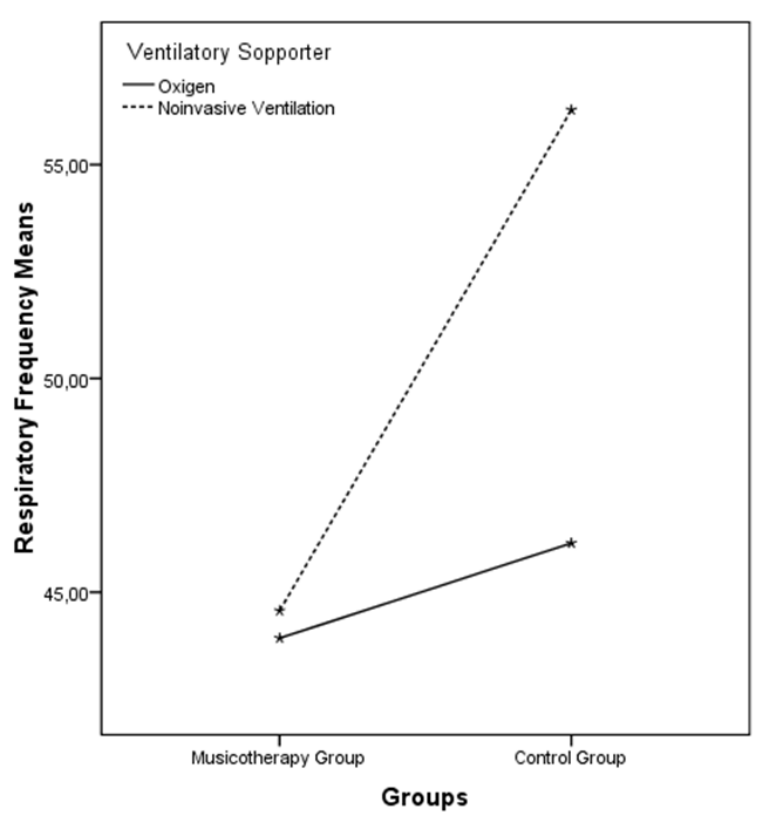

Figure 2: The interaction between music therapy and the ventilation support. The respiratory rate variation was lower in newborn of music therapy group with oxygen support. ( $F(5.06: \mathrm{p}: 0.01)$

\section{DISCUSSION}

Despite the non-equivalence of the statistical significance of the results, we observed clinical importance of music during the respiratory physiotherapy. In the studies we found that newborn infants on music therapy group showed clinically less variability of all physiological variables during the physiotherapy procedures. A clinical important result cannot always be considered statistically significant ${ }^{14}$.

This difference is particularly important considering the procedure performed in the two groups, it is considered invasive ${ }^{15}$. Studies report that, as compensatory mechanism to keep the cardiac output, the physiotherapy procedures applied to the chest, increase intrathoracic pressure and heart rate ${ }^{15-17}$.

Main $E^{15}$ showed some evidence that physiotherapy treatments had small but statistically distinguishable effects on respiratory function. The authors suggested that physiotherapy was more effective at reducing the airway resistance, presumably as a result of removing secretions, but also produced an increase in arterial blood gas markers for metabolic acidosis ${ }^{15}$.

Our findings differ from these studies, and such differences must reflect the inclusion of music as supportive to physiotherapy procedures.

Music can have a substantial effect in the modulating of affective states in hospitalized premature infants when dosed in a manner similar to medication as a therapeutic application for clients who are unable to express themselves in language ${ }^{16}$.

Thus, a therapeutic intervention for infants in the Neonatal Intensive Care Unit (NICU) and a special physiotherapy care that combines the infant's need with the demonstrated affective and physiological regulating qualities of music appears highly indicated and such an approach is available in sympathetic improvisational musictherapy ${ }^{17}$.

The music during therapeutic interventions appears to hold promise as a supportive sensory experience for the medically fragile newborn infant, and a range of positive sensory experiences such as contingent singing is necessary for healthy infant neurological development, may promote the social and neurological development of medically fragile newborn infants. Specifically, in the socially diminished environment of the NICU ${ }^{18,19}$.

In our results, the increase in heart and respiratory rate during the physiotherapy procedures only occurred in the group who did not receive music therapy during the procedures. Although there is not a good coordination between the respiratory muscles, the respiratory rate is more sensitive to peripheral stimuli and the baroreceptors are still immature in newborn infants ${ }^{20}$.

We found in our study that $30 \%$ the variation in respiratory rate occurred due to the inclusion of music during and after the physiotherapy and this result was even more evident when on mechanical ventilation.

The positive effects of music are cited in other articles that also evaluate the variability of physiological parameters on stressful procedures to newborn infants.

These studies show that music decreases heart rate 21-25 $^{2}$ nd increases oxygen saturation 2,26-29, and alteration of energy consumption and behavioral variables ${ }^{4,21,26}$ confirming data from this study.

In these articles ${ }^{2,4,21-29}$ there is a concern that the unfavorable environment of the NICU may compound a morbidity and the modification of the environment with the addition of music could minimize the iatrogenic effects.

The hypothesis is that music plays fundamental role on newborn infants' autonomic system, promoting a pituitary glandule stimulation, liberating endorphin and decreasing liberation of catecholamine, causing reduction of physiology parameters and lower behavioral variation ${ }^{4}$.

Other studies have shown that changes in behavioral state are fleeting and discrete after painful stimulus, making the behavioral evaluation hard and insufficient ${ }^{30}$. This perspective, physiological variables have contributed to the tracking of the pain and stress in newborn infants. These variables have been often used in other studies due to ease the application of this method, and being representative of the cardiopulmonary function and used routinely in neonatal unities ${ }^{9}$.

Although, Krueger $\mathrm{C}^{28}$ have stated that music therapy with the maternal voice develops better effect on adequacy behavioral state of newborn infants. We showed in our study that the sound of music on a tape recorder was enough to change clinically the evaluated physiological variables.

The tape recorder was also used by Chou $\mathrm{L}$ et $\mathrm{al}^{2}$. In their study, in order to evaluate the variation of oxygen saturation in preterm infants with and without music therapy during endotracheal 
aspiration procedure, the authors showed that music therapy has shown beneficial results in oxygen saturation, besides promoting decrease in heart rate and less variation in respiratory rate, corroborating the results in our study.

Even though our results show that the effects of music therapy promote less variation in respiratory rate and lower clinical variability of heart rate and oxygen saturation, we have not followed

\section{REFERENCES}

1. Yamanouchi I, Fukuhara H, Shimura Y. The transmission of ambient noise and selfgenerated sound in the human body. Acta Pediatr Jpn. 1990; 32(6): 615-24. DOI: http:/ /dx.doi.org/10.1111/j.1442-200X.1990. tb00895.x

2. Chou LL, Wang RH, Chen SJ, Pai L. Effects of music therapy on oxygen saturation in premature infants receiving endotracheal suctioning. J Nur Res. 2003;11(3): 209-16.

3. Batt-Rawden K, Tellnes G. How music may promote healthy behavior. Scand J Public Health. 2011; 39(2):113-20. DOI: http://dx. doi.org/10.1177/1403494810393555

4. Lubetzky R, Mimouni FB, Dollberg S, Reifen R, Ashbel G, Mandel D. Effect of music by mozart on energy expenditure in growing preterm infants. Pediatrics. 2010; 125(1): e24e28.DOI: http://dx.doi.org/10.1542/peds. 2009-0990

5. Olischar $M$, Shoemark $H$, Holton $T$, Weninger $M$, Hunt RW. The influence of music on aEEG activity in neurologically healthy newborns $\mathrm{e}^{\prime \prime}$ 32 week's gestational age. Acta Paediatr. 2011; 100(5): 670-75. DOI: http://dx.doi.org/10. 1111/j.1651-2227.2011.02171.x

6. Arnon S, Shapsa A, Forman L, Regev R, Bauer $\mathrm{S}$, Litmanovitz I, et al. Live music is beneficial to preterm infants in the neonatal intensive care unit environment. Birth. 2006; 33(2):131-6. DOI: http://dx.doi.org/10.1111/j.07307659.2006.00090.x

7. Field T, Hernandez-Reif M, Feijo L, Freedman J. Prenatal, perinatal and neonatal stimulation: a survey of neonatal nurseries. Infant Behav Dev. 2006; 29(1):24-31. DOI: http://dx.doi.org/ 10.1016/j.infbeh.2005.07.006

8. Standley J. Music therapy research in the NICU: an updated meta-analysis. Neonatal Netw. 2012; 31(5): 311-16. DOI: http://dx.doi.org/ 10.1891/0730-0832.31.5.311

9. Rahlin M, Stefani J. Effects of music on crying behavior of infants and toddlers during physical therapy intervention. Pediatr Phys Ther. 2009; 21(4):325-335. DOI: http://dx.doi.org/ 10.1097/PEP.0b013e3181bf00f7

10. Keith DR, Russel K, Weaver BS. The Effects of music listening on inconsolable crying in premature infants. J Music Ther. 2009; 46(3): 191-203. DOI: http://dx.doi.org/10.1093/jmt/ 46.3.191

11. Silva CM, Cação JMR, Silva KCS, Marques CF, Merey LSF. Respostas fisiológicas de recém- this long term effect and did not analyze the effect of live music and recorded music.

These limitations do not invalidate the results discussed, but we believe that further studies must be done.

The results suggest that there are beneficial effects of music, pointing lower respiratory rate in newborn infants during and after the respiratory physiotherapy.

nascidos pré-termo submetidos à musicoterapia clássica. Rev Paul Pediatr. 2013; 31(1):30-6. DOI: http://dx.doi.org/10.1590/ S0103-05822013000100006

12. Diniz NF, Gomes ELFD, Moran CA, Pereira SA, Martins LMA, Pereira LC. Assessment of the effects of manual chest compression technique on atelectasis in infants: a randomized clinical trial. Int J Clin Med. 2014; 5(9): 507-513. DOI: http://dx.doi.org/10.4236/ijcm.2014.59070

13. Pereira SA, Moran CA. Estimulação visual precoce para recém-nascidos prematuros internados na unidade neonatal. [cited 2015 Jun 18] Available from: http://www. moreirajrcom.br/revistas.asp?id_materia= 5856\&fase $=$ imprime.

14. Sola A, Dieppa FD, Rogido MR. An evident view of evidence-based practice in perinatal medicine: absence of evidence is not evidence of absence. J Pediatr. 2007; 83(5):395-414. DOI: http://dx.doi.org/10.2223/JPED.1702

15. Main E, Castle R, Newham D, Stocks J. Respiratory physiotherapy vs. suction: the effects on respiratory function in ventilated infants and children. Intensive Care Med. 2004; 30(6):1144-51. DOI: http://dx.doi.org/ 10.1007/s00134-004-2262-0

16. Trevarthen C, Malloch SN. The dance of wellbeing: defining the musical therapeutic effect. Nordic Journal of Music Therapy. 2000; 9(2): 3-17. DOI: http://dx.doi.org/10.1080/ 08098130009477996

17. Shoemark H. Contingent singing: The musicality of companionship with the hospitalized newborn infant. In: Baker F, Uhlig S. Therapeutic voicework in music therapy. 2011; p. 229-49.

18. Butt ML, Kisilevsky BS. Music modulates behavior of premature infants following heel lance. Can J Nurs Res. 2000; 31(4): 17-39.

19. Beebe B, Jaffe J, Lachmann F, Feldstein S, Crown $C$, Jasnow M. Systems models in development and psychoanalysis: The case of vocal rhythm coordination and attachment. Infant Mental Health J. 2000; 21(1-2): 99-122. DOI: http://dx.doi.org/10.1002/(SICI)10970355(200001/04)

20. Carroll JL. Invited review: developmental plasticity in respiratory control. J Appl Physiol. 2003; 94(1): 375-389 DOI: http://dx.doi.org/ 10.1152/japplphysiol.00809.2002

21. Hatem TP, Lira PIC, Mattos SS. The therapeutic effects of music in children following cardiac surgery. J Pediatr. 2006; 82(3): 186-92. DOI: http://dx.doi.org/10.2223/JPED.1473 
22. Yildiz A, Arikan D. The effects of giving pacifiers to premature infants and making them listen to lullabies on their transition period for total oral feeding and sucking success. J Clin Nurs. 2012; 21(5-6): 644-56. DOI: http://dx.doi.org/ 10.1111/j.1365-2702.2010.03634.x

23. O'Gorman S. Infant-Directed singing in neonatal and paediatric intensive care. Austr New Zealand J Family Ther. 2007; 28(2):100-8. DOI: http://dx.doi.org/10.1375/anft.28.2.100

24. Gilad E, Arnon S. The role of live music and singing as a stress-reducing modality in the neonatal intensive care unit environment. Music Med 2010; 2(1):18-22. DOI: http://dx.doi.org/ $10.1177 / 1943862109357070$

25. Lubetzky R, Mimouni FB, Dolberg S, et al. Effect of music by Mozart on energy expenditure in growing preterm infants. Pediatrics 2010; 125 (1): e24-8.

26. Aydin D, Yildiz S. Effect of classical music on stress among preterm infants in a neonatal intensive care unit. Health Med. 2012; 6(9): 3162-3168.

27. Malloch SS, Shoemark $H$, Èrnèec R, Newnham C, Paul C, et al. Music therapy with hospitalized infants - the art and science of communicative musicality. Infant Ment Health J. 2012; 33(4): 386-399. DOI: http://dx.doi.org/10.1002/ imhj. 21346

28. Krueger C. Exposure to maternal voice in preterm infants: a review. Adv Neonatal Care. 2010; 10(1):13-20. DOI: http://dx.doi.org/ 10.1097/ANC.0b013e3181cc3c69

29. Hodges AL, Wilson LL. Effects of music therapy on preterm infants in the neonatal intensive care unit. Altern Ther Health Med. 2010; 16(5): 72-73.

30. Johnston C, Campbell-Yeo M, Rich B, Whitley J, Filion F, Cogan J, et al. Therapeutic touch is not therapeutic for procedural pain in very preterm neonates: a randomized trial. Clin J Pain. 2013; 29(9): 824-9. DOI: http://dx.doi.org/10.1097/ AJP.0b013e3182757650 